\title{
Microbiocinosis Formation in Birds Kept in Captivity
}

\author{
Sh. Kakhramanova \\ Department of infectious and parasitic diseases, Ivanovo \\ State Agricultural Academy named after D.K. Belyaev \\ Ivanovo, Russia \\ aaabca@mail.ru
}

\section{A. Martynov}

Department of infectious and parasitic diseases, Ivanovo State Agricultural Academy named after D.K. Belyaev

Ivanovo, Russia

martynov.vet@mail.ru

\author{
V. Turkov \\ Department of infectious and parasitic diseases, Ivanovo \\ State Agricultural Academy named after D.K. Belyaev \\ Ivanovo, Russia \\ professor-turkov@yandex.ru
}

\author{
N. Yakimenko \\ Department of infectious and parasitic diseases, Ivanovo \\ State Agricultural Academy named after D.K. Belyaev \\ Ivanovo, Russia \\ ninayakimenko@rambler.ru \\ L. Kletikova \\ Department of infectious and parasitic diseases, Ivanovo \\ State Agricultural Academy named after D.K. Belyaev \\ Ivanovo, Russia \\ doktor_xxi@mail.ru

\section{Pronin} \\ Centre for Preclinical Research, Federal Centre for Animal \\ Health \\ Vladimir, Russia \\ proninvv63@mail.ru
}

\begin{abstract}
Keeping swans in captivity leads to changes in their microbal background. The study of mucous membranes of larynx and cloaca as well as bedding and pool water aiming at investigation of composition of both residential and pathogenic microflora in swans was carried out. For this purpose culturalbiochemical, morphological, hemolytic and tinctorial properties of the obtained material were studied using general biological methods with subsequent identification of isolated cultures by Berdgi determinant. Microorganisms referring to 7 genera were isolated from the mucous membranes of the birds. 6 species of microbes belonging to genera Echerichia, Salmonella and Staphylococcus inhabit the mucous membranes of larynx and cloaca of $100 \%$ of birds under study. Three species of Staphylococci were found on the mucous membranes of larynx in the case of $C$.olor and on the mucous membranes of cloaca in the case of C.bewickii (S. Epidermidis, S. Aureus and S. saprophyticus). $\beta$ - hemolytic streptococcus was foumd on the mucous membranes of larynx in $C$. bewickii. Proteus was present on the mucous membranes of larynx in C.olor and the mucous membranes of cloaca in $C$. bewickii. Penicillium and Aspergillus were found in lavages from the mucous membranes of larynx and cloaca correspondingly in C.olor. Microorganisms referring to genera Echerichia, Salmonella, Staphylococcus (St. albus and St. saprophyticus), Streptococcus ( $\beta$-hemolytic streptococcus), Bacillus, Mucor, Penicillium, Aspergillus were isolated from the bedding and Echerichia, Salmonella, Streptococcus ( $\gamma$ - and $\beta$ - streptococci) - from the pool water.
\end{abstract}

\section{Keywords—Swans, microorganisms, stress, contamination}

\section{INTRODUCTION}

After animal birth, a newly born organism begins to contact with different microorganisms entering its respiratory and digestive ways. Different bacteria, actinomycetes, mould and yeast fungi, mycoplasma and others settle from the air on mucous membranes of upper respiratory ways from nestlings with the first inhalation. Most of the microbes are situated in anterior parts of the respiratory tract decreasing in the number towards the deeper portions, the least number settling after the bifurcation of the trachea. In several hours after incubation, the gastroenteral tract of newborns is occupied by microbes from the eggshell and nest air $[1 ; 2 ; 3]$.

Microbic landscape depends on parents' microflora, feed, contamination of environmental objects and raising conditions. Microflora composition can change both qualitatively and quantitatively in the course of animal life [4].

Residential microflora includes lactobacteria as well as intestinal bacilli and rods, exercising antagonistic effect on pathogenic microorganisms. Lactobacteria ihabiting the intestine excrete ferments facilitating the digestion of proteins, fats and carbohydrates, as well as assimilation of 
microelements, and produce indispensable aminoacids and vitamins.

The conventionally pathogenic microflora is present in the organism of both healthy birds and those recovered from different diseases. Originally entering the organism, microorganisms are in symbiotic connection with macroorganisms creating a temporary immunological equilibrium [5]. Under stresses accompanied by general weakening of the organism and decreasing of its natural protective functions, "microbal pressure" grows leading to formation of autoinfection. The latter may either take an independent course causing mass losses among birds especially the young ones or act as a secondary process [6].

Microflora composition is richer in free-living birds than in those kept in artificial environment $[7 ; 8]$. The microbal background in swans [9] kept in captivity is of interest because of their close contact with other birds species.

Therefore, the aim of this study was the investigation of the composition of both residential and pathogenic microflora of respiratory and digestive organs in swans kept in captivity in Ivanovo, as well as environmental elements contamination.

\section{MATERIALS AND METHODS}

The investigation was carried out on the basis of the department of infectious and parasitic diseases, Ivanovo State Agricultural Academy named after D.K. Belyaev, Ivanovo, Russia in 2016.

The objects of the present study were three swans species: mute swan (Cygnusolor (Gmelin, 1789)) (6 representatives), tundra swan (Cygnusbewickii (Yarrell, 1830) (6 representatives) and blacknecked swan (Cygnusmelancoryphus (Molina, 1782)) (6 representatives) and birds environment.

Smears from the mucous membranes of larynx and cloaca, as well as lavages from bedding and pool water, served as the subject of investigation.

Cultural-biochemical, morphological, hemolytic and tinctorial properties of the material under study were investigated with the help of general microbiological methods with the following identification by Bergi determinator $[10 ; 11$; $12 ; 13]$.

Cultural-biochemical properties of the bacteria were studied on the meat-peptonic agar, meat-peptonic broth, Endo, Levin, Ploskirev, Garro, Wilson-Bler media as well as bismuthsulphid agar. Morphological properties were determined according to microorganisms' growth character in nutrient media. Hemolytical properties of the isolated organisms were investigated by incubation on bloody meat- peptonic agar, their tinctorial properties - by light microscope and Gram smear staining. Cultural-morphological properties of fungi were determined by colonies growth character on Chapek medium, as well as by light microscope.

\section{RESULTS AND DISCUSSION}

Microorganisms found on the mucous membranes of swans are presented in tables 1 and 2, figures $1-7$.
In the course of the study of microbic background of respiratory ways and digestive channel microorganisms referring to 7 genera were located, 6 inhabiting the larynx mucosis and equal amount of cloaca mucous membranes. Microorganisms of genera Escherichia (Rahn 1937), Salmonella (Rahn 1937), Staphylococcus (Rosenbach 1884), Streptococcus (Rosenbach 1884) were found on the mucous membranes of larynx and cloaca in $100 \%$ of examined birds.

Escherichia $u$ Salmonella were found in all the birds on the mucous membranes of both larynx and cesspool. Most of the microbes referring to these genera are either pathogenic or conditionally pathogenic and are capable of disease producing under resistance reduction. The aggregate of aetiological factors promoting stress formation is 10 times higher in birds kept in captivity [4] which can lead to disease formation in invasion of even conditionally pathogenic microflora against the background of immunity decreasing.

Staphylococcic microflora is represented by 3 genera: $S$. epidermidis- conditionally pathogenic staphylococcus exercising its pathogenic action under immuno-depressive conditions. According to Quinn (2011), it can cause infections in immuno-compromised humans and mouth cavity and larynx mucous inflamation in dogs [14]. S. Saprophyticus performes protective role inhabiting the mucous membranes is in fact the part of normal microflora. Still it can cause disbacteriosis excessively multiplying in the digestive tract.

$S$. aureus was found in mute swan probably causing pododermatitis of both legs and osteoarthritis of the left knee joint [15].

TABLE I. LARYNX MUCOUS MEMBRANES MICROFLORA COMPOSITION

\begin{tabular}{|c|c|c|c|}
\hline $\begin{array}{c}\text { Microorganisms } \\
\text { genera }\end{array}$ & $\begin{array}{c}\text { Birds } \\
\text { species }\end{array}$ & & \\
\hline & C. olor & C. bewickii & C. melancoryphus \\
\hline Escherichia & + & + & + \\
\hline Salmonella & + & + & + \\
\hline \multicolumn{4}{|l|}{ Staphylococcus: } \\
\hline S.epidermidis & + & + & + \\
\hline S. aureus & + & & \\
\hline S.saprophyticus & + & + & + \\
\hline \multicolumn{4}{|l|}{ Streptococcus } \\
\hline$\gamma$-hemolytic & + & & \\
\hline$\beta$ - hemolytic & & + & \\
\hline a-hemolytic & & & + \\
\hline Proteus & + & & \\
\hline Penicillium & + & & \\
\hline
\end{tabular}

Streptococci found in swans are represented by three groups. $\gamma$-hemolytic streptococcus is present on the mucous membranes of cloaca in C.olor and $C$. bewickii as well as on the mucous membranes of larynx excessively in C. olor. $\alpha-$ hemolytic streptococcus is present on the mucous membranes of cloaca in C. melancoryphus. 
TABLE II. CLOACA MUCOUS MEMBRANES MICROFLORA COMPOSITION

\begin{tabular}{|c|c|c|c|}
\hline $\begin{array}{c}\text { Microorganisms } \\
\text { genera }\end{array}$ & $\begin{array}{c}\text { Birds } \\
\text { species }\end{array}$ & & \\
\hline & C. olor & C. bewickii & C. melancoryphus \\
\hline Escherichia & + & + & + \\
\hline Salmonella & + & + & + \\
\hline \multicolumn{4}{|l|}{ Staphylococcus: } \\
\hline S. epidermidis & + & + & + \\
\hline S. aureus & & + & \\
\hline S. saprophyticus & + & + & + \\
\hline \multicolumn{4}{|l|}{ Streptococcus } \\
\hline$\gamma$ - hemolytic & + & + & \\
\hline$\alpha$ - hemolytic & & & + \\
\hline Proteus & & + & \\
\hline
\end{tabular}

$\beta$-hemolytic streptococcus was typified on the mucous membrane of C.bewickii larynx .

The conditionally pathogenic Proteus usual for intestinal microflora was isolated from the cloaca mucous membrane of C. bewickii. Microorganisms from genera Proteus (GustavHauser 1885) and Penicillium (Thom, 1906) were isolated from larynx mucous membranes of $C$. olor and microorganisms from genera Proteus and Aspergillus (Desm, 1894) were isolated from its cloaca mucous membranes.

Microorganisms referring to genera Escherichia, Salmonella, Staphylococcus (St.albus and St.saprophyticus), Streptococcus ( $\beta$ - hemolysis ), Bacillus (Ehrenberg 1835), Mucor (Fresen, 1850), Penicillium, Aspergillus were isolated from the bedding lavages during the environmental investigation.

Microorganisms, referring to genera Escherichia, Salmonella, Streptococcus ( $\gamma$ - u $\beta$-streptococcus) were isolated from the pool water.

The bedding was considered to be much more contaminated than the pool water, Mucor lacking on the mucous membranes of larynx and cloaca in swans.

\section{CONCLUSION}

It has been stated that swans living jointly in captivity have much similar mucous membranes microbic background of respiratory and digestive systems. The study proves that microorganisms of genera Escherichia, Salmonella, Staphylococcus, namely S. saprophyticus and S.epidermidis, Streptococcus ( $\gamma$ - and $\alpha$-hemolytic), are residential and typical of the microbal background of swans. The representatives of genera Aspergillus, Penicillium, Staphylococcus (S. aureus) and Streptococcus ( $\beta$ - hemolytic) are pathogenic or potentially pathogenic and are capable of producing visceral diseases as well as septic lesions. Besides, mycotoxins excreted by fungi can exercise immuno-depressing action in birds, leading to activation of the conditionally pathogenic microflora, as well as to growing of its concentration and displacement of residential microorganisms by pathogenic ones. Microorganisms inhabiting the mucous membranes of swans are present in bedding and pool water causing constant contamination of birds and following environmental pollution.

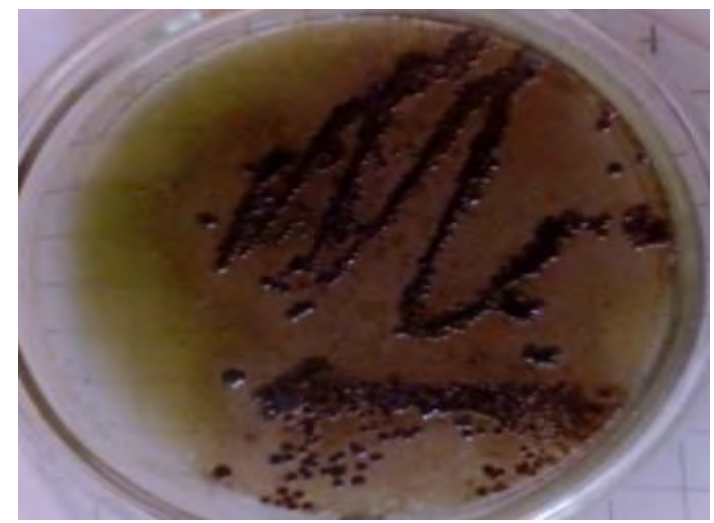

Fig. 1. Salmonella spp. Bismuth sulfite agar

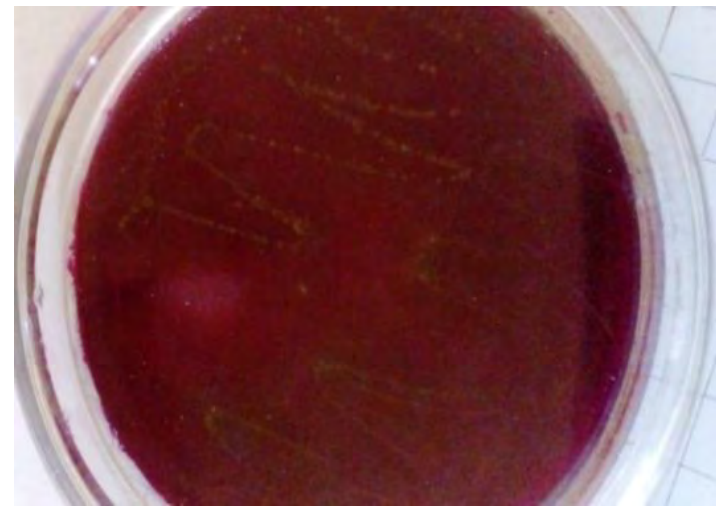

Fig. 2. E.coli. Nutrient medium Endo

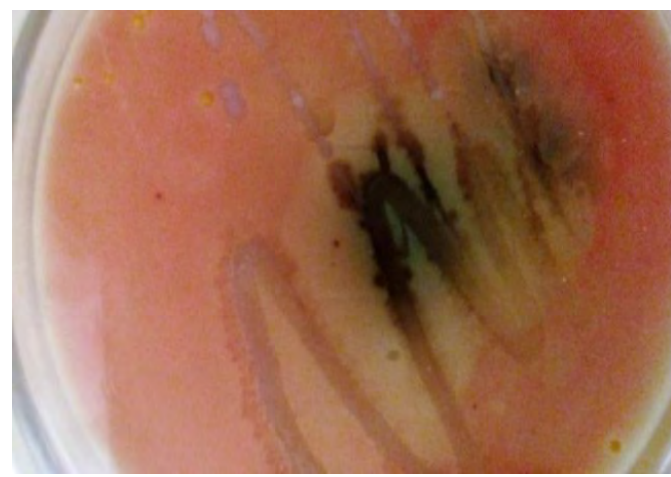

Fig. 3. . Streptococcus spp. Blood agar, $\alpha-, \beta$ - hemolysis

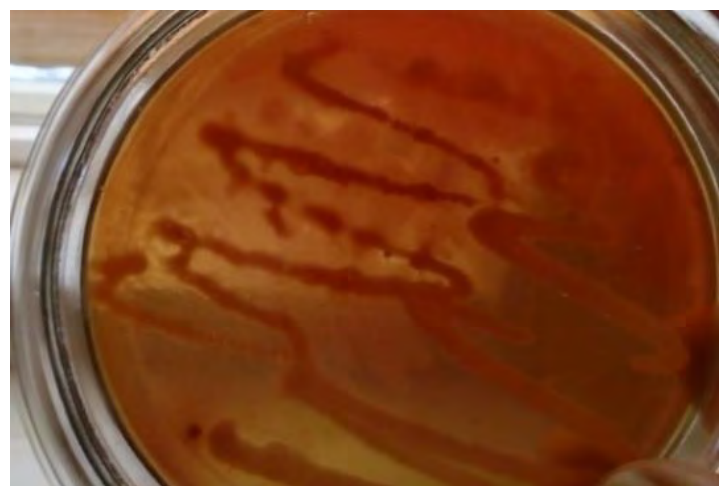

Fig. 4. Staphylococcus spp., Streptococcus spp. Blood agar, $\beta$ - hemolysis 


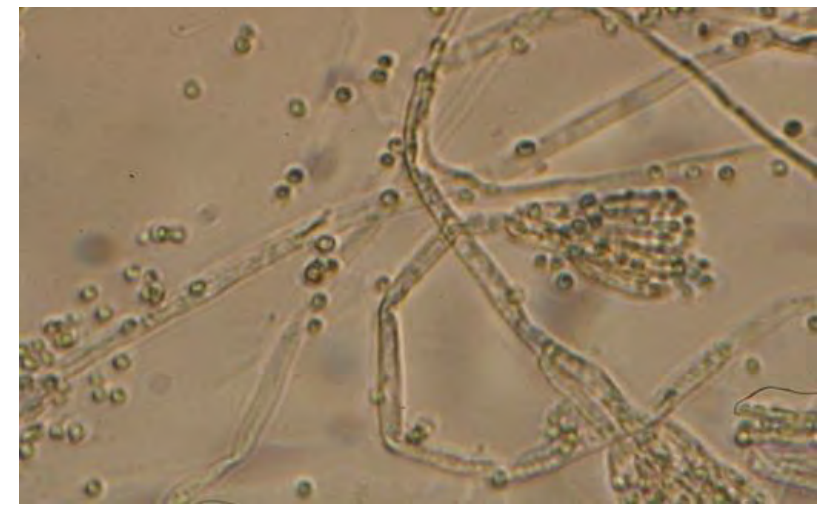

Fig. 5. Conidiophores and conidia. Penicillium spp. Nutrient medium of Capek

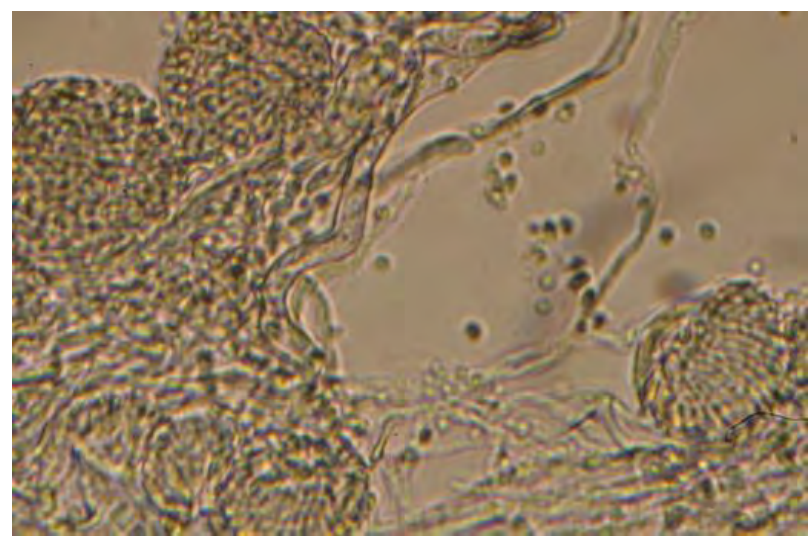

Fig. 6. Conidia and conidiophores Aspergillus fumigatus. Nutrient medium of Capek

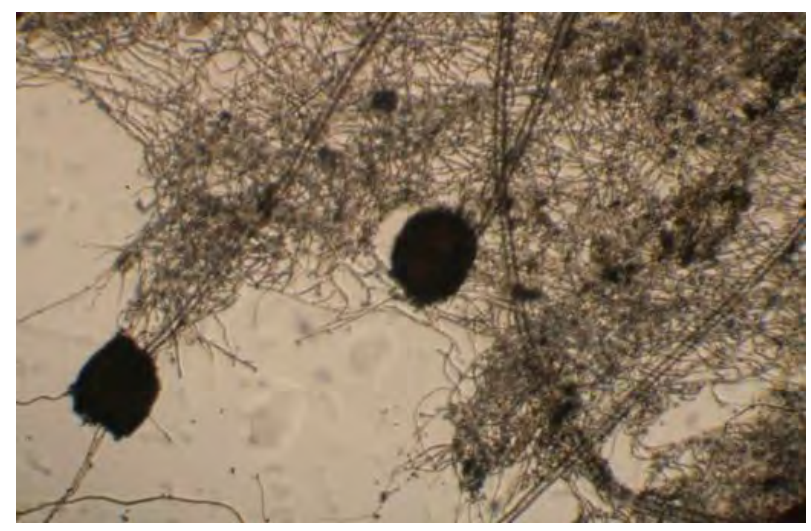

Fig. 7. Sporangiophores with sporangia. Mucor spp. Nutrient medium of Capek

\section{Acknowledgment}

The authors thank the rector of the Academy for his support.

\section{References}

[1] V.M. Bondarenko, T.V Matsulevich, Intestinal dysbacteriosis as clinicolaboratory syndrome: present state of the problem. Moscow, GEOTARMedia, 2007, pp. 292-304.

[2] Y.V. Lobzin, Y.P. Finogeev, V.M. Volzhanin, A.V. Semena, S.M. Zakharchenko, Infectious diseases: problems of adaptation. Saint Petersburg: ELBI-SPb, 2006, pp. 382-392.

[3] B.A Shenderov, "Medical microbic ecology: some research results and perspectives", Herald RAMS, 2005, vol. 12, pp. 13-17.

[4] V.G. Turkov, L.V. Kletikova, V.V. Pronin, V.A. Ponomarev, N.N Yakimenko, et al, Ecological and morpho-biochemical modifications of rock pigeon in anthropogenic landscapes. Ivanovo: PresSto, 2015, pp. 161-167.

[5] T.N Artemieva, Pathogenic and conditionally pathogenic hens' intestinal microflora and nontraditional antibacterial preparations efficiency, Saint Petersburg, 2004, pp. 12- 20.

[6] A.S. Oreshkin, V.V. Ponomarev, "The role of conditionally pathogenic microflora in young animals' diseases", Veterinary paper, 2000, vol. 20, pp. 3-4.

[7] T.I. Brezginova, D.Y. Kosterin, N.N. Yakimenko, Microflora of some wild birds of passage in Ivanovo region- Agrarian science in conditions of AIC modernization and innovational development, Ivanovo, 29 October 2015, pp. 11-13. [All-Russian research conference with international participation, 2015]

[8] L.V. Kletikova, N.N. Yakimenko, V.V. Pronin, V.A. Ponomarev, The results of biochemical swans blood study, Ekaterinburg, vol. 3, pp. 113116. [III International research-practical conference materials: State science in challenges epoch: past postulates and modern time theories]

[9] D. Diaz, Mycotoxins and mycotoxicoses, Moscow: Print city, 2006, pp. 275- 381 .

[10] A.K. Sarkisov, V.P. Koroleva, E.S. Kvashina, V.F. Grezin, Fungal diseases diagnostics (mycoses and mycotoxicoses) of animals. Moscow, Kolos, 1971, pp. 132- 144.

[11] M.A. Sidorov, D.I. Skorodumov, V.B. Fedotov, Zoopathogenic microorganisms determinant. Moscow, Kolos, 1995, pp. 310- 319.

[12] J. Hoult, Berdgi bacteria determinant. Moscow, Myr, 1997, pp. 375- 495.

[13] P.J. Quinn, B.K. Markey, F.C. Leonard, P. Hartigan, S. Fanning, E. S. FitzPatrick, Veterinary Microbiology and Microbial Disease. WileyBlackwell, 2011, pp. 45- 123.

[14] Sh. F. Kakhramanova, V.M. Khozina, L.V. Kletikova, V.V. Pronin, N.N. Yakimenko, V.N. Kokurin, A.N. Martynov, "Mute swans clinical examination in Ivanovo Zoo in 2015, Hypology and veterinary, 2015, vol. 4(18), pp. 22-27. 